IDDF2018-ABS-0085 PROSPECTIVE CROSS-SECTIONAL STUDY ON THE CLINICAL PROFILE AND MYOCARDIAL FUNCTION OF SEVERELY AND MODERATELY MALNOURISHED CHILDREN 2 MONTHS TO 18 YEARS OLD IN PHILIPPINE CHILDREN'S MEDICAL CENTRE (PCMC)

Odessa Bayani*. Philippine Children's Medical Center, Philippines

\subsection{6/gutjnl-2018-IDDFabstracts.7}

Background Malnutrition in children produces visceral protein loss and consequent cardiac atrophy. There are conflicting studies if this cardiac atrophy results in myocardial dysfunction.

This study aims to determine the prevalence of myocardial dysfunction and describe the demographic, clinical, electrolyte changes, electrocardiographic and structural and myocardial function in severely and moderately malnourished children.

Methods This is a prospective cross-sectional study conducted on severely and moderately malnourished children 2 months to 18 years old in Philippine Children's Medical Centre. Serum potassium, magnesium, ionised calcium and phosphorus determination, 2D echocardiography and electrocardiogram (ECG) were done.

Results 73 subjects (43 moderately and 30 severely malnourished) were recruited. Majority had neurologic diseases $(n=18$, $25 \%)$, followed by tuberculosis $(n=13,18 \%) .2$ had pneumonia, 2 had diarrhoea.

Hypokalemia was found in $5 / 30$ or $16 \%$ severely malnourished (Potassium 2.21-2.96 mmol/L) and $4 / 42$ or $9 \%$ moderately malnourished (Potassium 2.5-2.89 mmol/L) ( $\mathrm{p}>0.05)$. Only one marasmic child had hypomagnesemia $(0.5 \mathrm{mmol} / \mathrm{L})$. Ionised calcium and phosphorus were normal. Both groups demonstrated prolonged QT interval, short and prolonged PR interval, prolonged QRS duration with no statistical significance. The most common ECG finding is short PR interval in moderately $(n=29 / 42$ or $69 \%)$ and severely malnourished $(\mathrm{n}=18 / 29$ or $62 \%)$ subjects. $(\mathrm{p}>0.05)$

The systolic and diastolic functions were normal in both the severely and moderately malnourished. Although the left ventricular mass (LVM) was low with no statistical significance between the two groups, the LVM index remained to be normal. The presence of pericardial effusion in severely malnourished $(n=5 / 29,17 \%)$ compared with only 1 in moderately malnourished had a statistical significance of $\mathrm{P}$.

Conclusions Hypokalemia, hypomagnesemia and shortened PR interval are common in malnutrition. Hence, vigilant monitoring of electrolytes and ECG should be done in all malnourished children. Even if the left ventricular mass was reduced in malnutrition, the left ventricular mass index remained to be normal. No myocardial dysfunction was found in the moderately and severely malnourished children. This suggests a possible compensatory mechanism of a malnourished heart.

\section{IDDF2018-ABS-0094 HYPERPROLACTINEMIA, BEWARE OF PROKINETIC DRUGS!}

Unknown Shivam*, Atluri Sridevi. Vydehi Institute of Medical Sciences and Research Center, India

10.1136/gutjnl-2018-IDDFabstracts.8
Background Hyperprolactinemia is the most common cause of galactorrhoea in women. Levosulpride an atypical neuroleptic and a prokinetic drug acting only on the D2 receptors can cause significant hyperprolactinemia which may be associated with galactorrhea. However, it is often under- or misdiagnosed. Hence, we describe a case of drug induced hyperprolactinemia presenting with galactorrhoea.

Methods A 31 years old lady presented with complains of menstrual irregularity and galactorrhoea since 18 months. She consulted an Endocrinologist in the USA who started her on Cabergoline $0.25 \mathrm{mg}$ twice a week. However, galactorrhoea did not subside. MRI of the pituitary gland was unremarkable. Cabergoline dose was increased to $0.5 \mathrm{mg}$ twice a week and then gradually to $2 \mathrm{mg}$ per week. After few months of treatment, she was shifted to Bromocriptine in view of persistent galactorrhea and hyperprolactinemia not responding to cabergoline. She presented to us in April 2017 with unabated symptoms and elevated serum prolactin $(152 \mathrm{ng} / \mathrm{ml})$. A review of drug history revealed intake of Esomeprazole $(40 \mathrm{mg})$ and Levosulpride $(75 \mathrm{mg}$ ) combination pill since January 2016 for dyspepsia. Levosulpride induced hyperprolactinemia was suspected and hence it was stopped.

Results After a week of stopping levosulpride serum prolactin level reduced to $13.48 \mathrm{ng} / \mathrm{ml}$ whereas galactorrhea subsided completely. These findings confirmed levosulpride induced hyperprolactinemia.

Conclusions We have made significant advances in the field of medicine, but this case teaches us to never forget the basics of medicine - A detailed history and clinical examination. Careful drug history and awareness about levosulpiride and its effect on serum prolactin levels would avoid unnecessary costly investigations and treatment.

\section{IDDF2018-ABS-0096 MAJOR GENETIC ALTERATIONS IN SPORADIC COLORECTAL CANCER IN THE CHINESE POPULATION}

Po Man Tsang*. The Chinese University of Hong Kong, Hong Kong

10.1136/gutjnl-2018-IDDFabstracts.9

Background Sporadic colorectal cancer accounts for $80 \%$ of all colorectal cancer. The 2 major mechanisms of genetic alteration are chromosomal instability (CIN) and microsatellite instability (MSI). The prevalence of KRAS, NRAS, BRAF and CTNNB1 mutations in western population was 42.4\%, 5.1\%, $15.2 \%$ and $1.1 \%$. BRAF mutations in Chinese population were much lower (3.1\%). RNF43 mutation was recently reported with a high prevalence of $18 \%$. It was found mutually exclusive with APC inactivation and was associated with MSI-H tumours.

In this study, the prevalence of APC, KRAS, NRAS, BRAF, CTNNB1 and RNF43 mutations in Chinese sporadic colorectal cancer patients was confirmed by next generation sequencing (NGS). The association of RNF43 mutation with APC, BRAF mutations and MSI status of tumours, was analysed. Clinical-pathological correlation was evaluated.

Methods NGS was used to investigate the prevalence of major mutations in 55 subject samples. Data analysis was done by Statistical Package of the Social Sciences (SPSS) statistics. Clinical-pathological correlation was analysed by Cox regression and Kaplan-Meier estimate. 